\title{
Variation Feature and Fuzzy Comprehensive Evaluation about Earthquake-damaged Environment Hazard for Underground Structure
}

\author{
Fengshan Wang, Hui Yuan, Bo Wang, Wanhong Zhu and Houqing Lu \\ College of Field Engineering, PLA University of Science \&Technology, Nanjing 210007, China \\ E-mail:WFS919@126.COM
}

\begin{abstract}
Keywords: underground structure; earthquake-damaged environment; hazard; variation coefficient; fuzzy comprehensive evaluation; Entropy

Abstract: According to subjective deviation and variation characteristics in fuzzy decision-making, one fuzzy reasoning method is put forward on the environment hazard for earthquake-damaged underground structure with the integration of entropy and variation weight distribution. Through identifying such index system and the internal fuzzy quantitative relation within the environment hazard of earthquake-damaged underground engineering, the fuzzy comprehensive decision-making matrix is given, the entropy weight and variation weight is calculated for the above index, which is dynamically revised by distribution coefficient, and erects the fuzzy comprehensive evaluation model for the risk monitoring unit; Through composing the above fuzzy comprehensive evaluation and the similarity vector, the earthquake-damaged environment hazard is entirely measured, and the risk law is gained for such hazard. Case shows that model effectively solves the earthquake-damaged environment risk decision-making problem for underground structure, which provides a theory and method for the scientific reliefs and practice decisions.
\end{abstract}

\section{Introduction}

Under the complex earthquake effects, the regional topography transformations, building collapse, land rise and subsidence, mountain crack, destruction and interruption of water supply facilities, debris accumulation, mountains and rivers diversion, road twists and slumping, and other damage phenomena, results in the wicked and frailty environment conditions around the underground structure [1]. Therefore, how to timely, efficiently and orderly control the complex earthquake-damaged environment around the underground structure, to scientifically discover and hold in the up growth characteristics and evolvement disciplinarian about earthquake-damaged environment, presents an important value for disaster reconstruction and prevention [2].

Repeated impact of the earthquake and aftershocks, and the instant superposition and attenuation of outside load, leads to the anabatic transition in the geology, engineering, water environment and other aspects [3]. The various evolutions in surface sliding body, tunnel cracks, surrounding rock seepage and other conditions, is in a certain highly sensitive period to the several outside elements, whose risk lacks one accurate disaster chain, and this is a complex complicated systematic engineering, with multi-factor, multi-level, multi-coupling, uncertainties and other characteristics [4]. For the simple model, good applicability, the excellent evaluation effectiveness to complex issues, and the good coupling nature with other algorithms or models, Fuzzy Theory has been widely used in the risk natural disaster evaluation operations [5], such as the optimized classification and application of surrounding rock based on fuzzy analytic hierarchy process [6], the fuzzy multiple criteria decision-making approach for Taiwan railway reconstruction risk assessing operations [7].

The unintelligibility in measuring the index standards and boundaries, and the subjectivity in the whole fuzzy evaluating process, could easily lead to the deviation from the evaluation outcome to the impersonal fact, which is one important problem in influencing the feasibility and reliability of Fuzzy Comprehensive Evaluation Method [8]. At home and abroad, many scholars give the more reasonable solution for the fuzzy mathematic application, through the index weighting, improvements in the decision-making criteria, and other methods. For example, the interval-valued intuitionistic fuzzy principal component analysis model is applied into the complex multi-attribute large-group 
decision-making operation [9], and a modified fuzzy min-max neural network is applied into the fault detection and classification [10].

Adapting to the complex characteristics and the variant evolution features in earthquake-damaged environment around underground structure, and breaking the traditional understanding and application about Linear Fuzzy Comprehensive Evaluation, the fuzzy transformation is applied into the domain conversion on the fuzzy concepts of the earthquake-damaged risk environment, which systematically evaluates this issue. This method enriches and expands the multiple variables and evidences coordination and matching applications of Fuzzy Comprehensive Evaluation in the complex decision-making problems, which shows the certain practical significance and valuable applications.

\section{Hazard Evaluation Index System on Earthquake-damaged Environment}

Principles in Hazard Evaluation Index System Construction. How to unbiased select the index, and establish the index system for environment hazard around earthquake-damaged underground structure, has directly impact on the risk decision-making accuracy and reliability, which could provide the solid foundation for the environment Hazard evaluation around underground structure.

Up to now, there is no the authoritative, uniform and normative evaluation index system for the earthquake-damaged risk environment around underground structure [11]. The selecting and designing process for the Hazard evaluation index system, must focus on the representative meaning of each index, and focus on the internal structure function of the hazard index system. To scientifically realize the index abstracting process by rule and line, the following basic principles must be observed.

(1)Comprehensive and Complete Principle. All the index must take on the fairly standard scientific explanation, and the index system should fully and objectively reflect the basic connotation and characteristics about earthquake-damaged risk environment around underground structure.

(2) Clear Arrangement Principle. The construction about the Hazard evaluation index system should accurately reflect the dominance relationship among the various indexes, build up the index macrocosm with the clear arrangement and logical structure, and eliminate the compatibility within the index system, to ensure that scientific evaluation.

(3)Concise Science Principle. The Hazard evaluation index System must be scientific, concise, clear, logical meaning, and the system logical structure must take on the maximum inclusiveness, which doesn't only contain the risk aspects and all contents, but also pay attention to keep a lookout on the conflict within the index representativeness and simplicity [12].

Earthquake-damaged Hazard Evaluation Index System Constitution. Following the above index selecting and designing principles on environment hazard around earthquake-damaged underground structure, centering on the variant elements in the geological environment conditions, the multiple features, characteristics, indicators and other kinds factors, especially the overlapping information should be fully abstracted. In accordance with the logical judgment process of "Specific - Abstract Specific", the essential characteristics are recognized with the gradual deepening, fine and systematic procedure.

Then, the scientific, concise index system is established for certain environment hazard evaluation around the earthquake-damaged underground structure, including Terrain and Landform Elevation Variation, Regional Slope Variation, Height variation, Rock Structural Variation, Geological Structural Surface Intensity Variation, Land Subsidence Cracks Scale, Grounder water Seepage Discharge, Grounder water Resources Intensity Variation, Building Damage Hazard Severity, Geology Environment Pollution Degree, Pore Water Pressure Variation, Landslide Body Weight.

Hazard Evaluation Index System, totally include the above 12 piece of index. Accordingly, $C_{j}$ is denoted as the common index symbol for the Hazard evaluation index, and the set is $C=\left\{C_{1}, C_{2}, \ldots C_{n}\right\}$. 
Mathematical Expression and Fuzzy Quantification on Hazard Evaluation Index. Index set and reviews set are the two prerequisite in the application of Fuzzy Comprehensive Evaluation Method [12]. The reviews set is expressed as $V=\left\{V_{\mathrm{I}}, V_{\mathrm{II}}, V_{\mathrm{III}}, V_{\mathrm{IV}}, V_{\mathrm{V}}\right\}$, namely 5 evaluation grade, and the language mode is expressed as \{the worst risk, worse risk, relative risk, lower risk, lowest risk\},

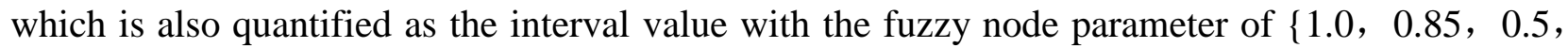
$0.35,0\}$.

Under the fuzzy quantifying reviews from the decision makers about the individual hazard index of $C_{j}$, namely the membership degree of $C_{j}$ to each evaluation grade, the fuzzy mapping relation is established for the evaluation operation, as shown in Equation (1).

$$
\left\{\begin{aligned}
f: u & \rightarrow F(V) \\
C_{j} & \text { a } f\left(C_{j}\right)=\frac{x_{1}^{j}}{V_{1}}+\ldots+\frac{x_{k}^{j}}{V_{k}}+\ldots+\frac{x_{\mathrm{V}}^{j}}{V_{\mathrm{V}}}
\end{aligned}\right.
$$

Thus, in accordance with Equation (1), $\alpha$ is set as the fuzzy hazard evaluation for the observed objects to each index of $C_{j}$, and $M(\cdot, \oplus)$ denotes the fuzzy hazard calculation operators, namely $\alpha=\sum_{k=1}^{p} x_{k}^{j} \cdot V_{k}$.

\section{Environment Hazard Evaluation Index Weights}

Fuzzy Decision-making Matrix on Environment Hazard Evaluation. Set $u_{1}, u_{2}, u_{3}, \ldots, u_{n}$ as the risk observed objects, and $u_{i}$ as the common symbol of the the risk observed object, which is also called as the monitoring unit or risk evidence body; The set of $u_{i}$ is denoted as $\Theta$ for the risk evidence framework about the environment Hazard around earthquake-damaged underground structure.

According to the fuzzy mapping relation expression of Equation (1), and the inherent fuzzy calculation method, $x_{i j}$ is denoted as the fuzzy reckoning measurement for the monitoring unit of $u_{i}$ under the hazard evaluation index of $C_{j}$. As the elements varies in the matrix of $\left[x_{i j}\right]_{m \times n}$, the risk decision-making result would be directly attested for the environment hazard evaluation; And, some extreme data within the sample space would be balanced and weakened. To enlarge the value effect of the observed objects, $u^{+}$and $u^{-}$is designed as the virtual sample with the corresponding greatest and lowest risk condition among the monitoring samples.

Let $u^{+}$as the virtual sample symbol with the greatest risk for the earthquake-damaged environment, and this value step is $\left.u^{+}=\left\{x_{m+1, j} \mid 1 \leq i \leq m\right)\right\}$; on the contrary, $u^{-}$is denoted as the virtual sample symbol with the lowest risk, and that value step is $\left.u^{-}=\left\{x_{m+2, j} \mid 1 \leq i \leq m\right)\right\}$. The value of the element in $u^{+}$and $u^{-}$could be obtained with the following calculation mode, as shown in Equation (2).

$$
\left\{\begin{array}{l}
x_{m+1, j}=\max _{1 \leq i \leq m}\left\{x_{i j}\right\}(j=1,2, \ldots, n) \\
x_{m+2, j}=\min _{1 \leq i \leq m}\left\{x_{i j}\right\}(j=1,2, \ldots, n)
\end{array}\right.
$$

Accordingly, the fuzzy calculation information and the extreme changes could be concluded about the risk observed objects, and the fuzzy decision-making matrix is established on the hazard evaluation operation for earthquake-damaged environment around underground structure, namely $X=\left\{x_{i j}\right\}_{(m+2) \times n}$.

Calculate the Entropy Weight on Hazard Environment Evaluation. To avoid the evaluation deviations caused by the subjective factors, the entropy method is applied into determining the index weights for the earthquake-damaged environment hazard evaluation. The entropy measurement to the disorder degree in the decision-making system, reduces the complexity and uncertainty, which provides the relative objective index weight for certain risk evaluation operation [13]. 
To normalize the fuzzy decision-making matrix of $X, y_{i j}$ is denoted as the proportion of the observed object of $u_{i}$ under the index of $C_{j}$. And, the reckoning method is $y_{i, j}=x_{i, j} / \sum_{i=1}^{m} x_{i j}$, namely Normalization Step.

In accordance with the reckoning method of Information Entropy Theory [13], the Shannon entropy could be obtained through Equation (3).

$$
e_{j}=H\left(C_{j}\right)=-\frac{1}{\ln (m)} \sum_{i=1}^{m} y_{i j} \ln \left(y_{i j}\right)
$$

In Equation (3), $e_{j}$ denotes the information entropy value of $C_{j}$; As $e_{j}$ shows the greater value, the greater internal disorder degree exits in the risk evaluation characteristics; And if $y_{i j}=0, y_{i j}=0.00001$ should replace this worse situation.

At this point, the entropy weight of $C_{j}$ could be obtained through the following calculation procedure, as shown in Equation (4).

$$
\theta_{j}=\left(1-e_{j}\right) /\left(n-\sum_{j=1}^{n} e_{j}\right), \quad \sum_{j=1}^{n} u_{j}=1
$$

In Equation (4), $\theta_{j}$ shows the weight $C_{j}$, and $\tilde{\theta}$ shows the weight set, namely $\tilde{\theta}=\left\{\theta_{j} \mid j=1,2, \ldots, n\right\}$, for the environment hazard evaluation about earthquake-damaged underground structure.

Calculate the Variation Weight on Hazard Environment Evaluation. Although the entropy weighting method could objectively reflect the index weights about the hazard evaluation issue for earthquake-damaged underground structure, this method seriously depends on the initial matrix, which hardly reflects the variability in the environment hazard evaluation system. Therefore, Variation Coefficient Method could be applied into the index weighting program for the environment hazard evaluation [14], and finally Distribution Coefficient is applied into the index weight combination between the entropy weights and the variation weights.

Three point estimating method is applied to the average calculation, namely determining the average value for the individual index of $C_{j}$ under the risk evidence framework of $\Theta$. And, $\overline{x_{j}}$ is the average value symbol, as in Equation (5).

$$
\overline{x_{j}}=\frac{1}{6}\left(x_{m+1, j}+x_{m+2, j}+\frac{4}{m} \sum_{i=1}^{m} x_{i j}\right)
$$

With the average value of $\overline{x_{j}}$, there is various deviation in the sample data space of $X$. And, the deviation degree of $C_{j}$ should be acquainted with the observed object framework of $\Theta$, as $\operatorname{Var}\left(u_{j}\right)$.

$$
\operatorname{Var}\left(u_{j}\right)=\sqrt{\frac{1}{m-1} \sum_{i=1}^{m}\left(x_{i j}-\overline{x_{j}}\right)^{2}}
$$

Here, $\operatorname{Var}\left(u_{j}\right)$ denotes the deviation degree of $C_{j}$. With the deviation measurement of $\operatorname{Var}\left(u_{j}\right)$, the variation degree of $C_{j}$ could be reckoned, which is used to denote the index weight under the variation coefficient condition, as shown in Equation (7).

$$
\tau_{j}=\operatorname{Var}\left(u_{j}\right) / \overline{x_{j}}, \quad \sigma_{j}=\tau_{j} / \sum_{j=1}^{n} \tau_{j}
$$

Here, $\tau_{j}$ is th intermediate variable to determine the index weight with the variation coefficient, and $\delta_{j}$ denotes the index weight with the variation coefficient for the above environment hazard evaluation operation.

Weights Distribution on Hazard Evaluation Index. Combination Weighting Method could integrate the advantages of the single index weighting method, and avoid the sidedness of the single index weighting result [15]. The weight distribution coefficient is applied into the weighting 
combiation with the entropy weights and the variation weights. For the certain earthquake-damaged environment evaluation issue, $\Delta \phi$ is set as the weight distribution coefficient to combined $\theta_{j}$ and $\sigma_{j}$, and $0<\Delta \phi<1$ is the given limitation qualification, as shown in Equation (8).

$$
\bar{\varpi}_{j}=\Delta \phi \cdot \theta_{j}+(1-\Delta \phi) \cdot \sigma_{j}, \quad 0<\Delta \phi<1
$$

Here, $\Phi_{j}$ is the dynamic revised weight of $C_{j}$, reflecting the entropy weight information and the variation weight information, namely providing with all the subjective and objective aspects. The variety of $\Delta \phi$ embodies the variant features with the hazard index weighting connotations, and $\tilde{\omega}$ is the index weight coefficient vector for this environment hazard evaluation.

$\Delta \phi$ is the random value and the catastrophe value under the difference variation law of the hazard index weights. In order to meet the consistency between the difference degree among the different weights and the distribution coefficient, the constraint prerequisite is established for the distribution coefficient, namely the consistency of the distance function and the distribution coefficient, as shown in Equation (9).

$$
d(\tilde{\theta}, \tilde{\sigma})=|1-2 \cdot \Delta \phi|=\left[0.5 \sum_{j=1}^{n}\left(\theta_{j}-\sigma_{j}\right)^{2}\right]^{0.5}
$$

Here, $d(\tilde{\theta}, \tilde{\sigma})$ represents the distance function between the entropy weight of $\tilde{\theta}$ and the variation weight of $\tilde{\sigma}$ for the hazard index weighting combination about the earthquake-damaged environment around underground structure region.

\section{Fuzzy Comprehensive Evaluation Model on Environment Hazard}

Fuzzy Evaluation on Monitoring Units of Earthquake-damaged Environment. The fuzzy risk calculation operator of $M(\cdot, \oplus)$ is put into the practice, and $G$ is set as the fuzzy calculation vector under the monitoring unit framework of $\Theta$ about the earthquake-damaged underground structure environment, $G=\sum_{m}^{i=1} \sum_{n}^{j=1} x_{i j} \Phi_{j}$.

Here, $G=\left[\begin{array}{llll}g_{1} & g_{2} & \mathrm{~L} & g_{m}\end{array}\right]$, the element of $g_{i}$ denotes the risk fuzzy decision-making result for the earthquake-damaged environment evidence sample of $u_{i}$ in the certain underground structure. Similarity and Support Degree with Monitoring Units for Environment Hazard Evaluation. Reference and application of the evidence body concept in Dempster Shafter Evidence Theory [16], $u_{1}$ and $u_{2}$ is designed as the common two evidence body or monitoring unit in the risk environment decision-making framework of $\Theta$, and the corresponding index value vector is $x_{1 j}$ and $x_{2 j}$. The similarity coefficient of $u_{1}$ and $u_{2}$ could be obtained from the following expression, in Equation (10).

$$
\operatorname{Sim}_{12}=\frac{\sum x_{1 j} x_{2 j}}{\sqrt{\left[\sum x_{1 j}^{2}\right]\left[\sum x_{2 j}^{2}\right]}}
$$

Here, the similarity coefficient of $\operatorname{Sim}_{12}$, expresses the mutual support and depended within the risk evidence framework, and the range is $\operatorname{Sim}_{12} \in[0,1]$. As the greater value of $\operatorname{Sim}_{12}$, the higher support degree in the two evidence body.

In the risk evidence framework of $\Theta$ about the earthquake-damaged underground structure environment, there is $m$ piece of evidence body. With Equation (10), the similarity coefficient of $\operatorname{Sim}_{i j}$ could be reckoned out for the risk evidence of $u_{i}$ and $u_{j}$, which forms the similarity matrix of $\operatorname{Sim}, \operatorname{Sim}=\left\{\operatorname{Sim}_{i j}\right\}_{m \times m}$.

Add all the row vector of Sim, the support of all the risk framework to the certain evidence is $\operatorname{Sup}\left(u_{i}\right)=\sum_{j=1}^{m} \operatorname{Sim}_{i j},(i, j=1,2, \ldots, n)$. The certain evidence body is assigned as the key evidence entity with the greater support degree [16], and the selecting process is $\operatorname{Sup}(*)=\max \left\{\operatorname{Sup}\left(u_{i}\right)\right\}$. Here, $\operatorname{Sup}(*)$ is 
the key evidence entity. With $\operatorname{Sup}\left({ }^{*}\right)$ and $\operatorname{Sup}\left(u_{i}\right)$, the credibility of $\operatorname{Cre}\left(u_{i}\right)$ is obtained for the certain risk evidence body of $u_{i}$, which is abbreviated as $\mathrm{Cre}_{i}$, reflecting the whole support proportion within the overall risk evidence body. And, the reckoning process of $\mathrm{Cre}_{i}$ is the certain ratio calculation of $\operatorname{Sup}(*)$ and $\operatorname{Sup}\left(u_{i}\right)$, as $\operatorname{Cre}_{i}=\operatorname{Sup}\left(u_{i}\right) / \operatorname{Sup}(*)$.

Fuzzy Evaluation Operation on Environment Hazard. According to the risk measurement and credibility of each evidence body, the overall hazard evaluation could be reasoned through the following expression in Equation (11).

$$
H=G^{T} \cdot \tilde{S}^{T}=\left[\begin{array}{llll}
g_{1} & g_{2} & \mathrm{~L} & g_{m}
\end{array}\right] \cdot\left[\begin{array}{llll}
\text { Gre }_{1} & \text { Gre }_{2} & \mathrm{~L} & \text { Gre }_{m}
\end{array}\right]^{T}
$$

In Equation (11), $H$ expresses the overall evaluation on the environment hazard in the earthquake-damaged underground structure region. In the variation range of $\Delta \phi$, if $H$ shows the lower variation extent, the environment hazard could be sentenced into one accurate risk grade according to the fuzzy membership degree.

\section{Example Analysis}

Through the risk survey about the earthquake-damaged environment in some typical underground structures in Wenchuan Earthquakes as the background, the risk fuzzy decision-making is erected as one example for this model experiment by the decision-making groups, as shown in Tab.1. There is 6 piece of the risk sample, as the following $u_{1}, u_{2}, u_{3}, u_{4}, u_{5}$ and $u_{6}$.

Table 1 Fuzzy decision-making matrix on earthquake-damaged environment

\begin{tabular}{|c|c|c|c|c|c|c|c|c|c|c|c|c|}
\hline & $C_{1}$ & $C_{2}$ & $C_{3}$ & $C_{4}$ & $C_{5}$ & $C_{6}$ & $C_{7}$ & $C_{8}$ & $C_{9}$ & $C_{10}$ & $C_{11}$ & $C_{12}$ \\
\hline$u_{1}$ & 0.754 & 0.263 & 0.961 & 0.869 & 0.049 & 0.942 & 0.337 & 0.452 & 0.801 & 0.401 & 0.511 & 0.243 \\
\hline$u_{2}$ & 0.779 & 0.689 & 0.774 & 0.549 & 0.944 & 0.575 & 0.794 & 0.538 & 0.916 & 0.239 & 0.389 & 0.649 \\
\hline$u_{3}$ & 0.934 & 0.748 & 0.817 & 0.145 & 0.490 & 0.059 & 0.311 & 0.996 & 0.181 & 0.923 & 0.241 & 0.731 \\
\hline$u_{4}$ & 0.129 & 0.459 & 0.868 & 0.853 & 0.489 & 0.834 & 0.928 & 0.478 & 0.263 & 0.183 & 0.403 & 0.647 \\
\hline$u_{5}$ & 0.568 & 0.583 & 0.084 & 0.962 & 0.337 & 0.353 & 0.165 & 0.442 & 0.145 & 0.242 & 0.906 & 0.450 \\
\hline$u_{6}$ & 0.469 & 0.829 & 0.399 & 0.351 & 0.902 & 0.821 & 0.411 & 0.913 & 0.259 & 0.513 & 0.369 & 0.815 \\
\hline
\end{tabular}

The entropy value, entropy weight and variation weight could be reckoned for the certain risk index to the risk evaluation example, in accordance with Equation (4) and (7), as shown in Tab.2.

Table 2 Entropy and Variation weights of environment hazard evaluation index system

\begin{tabular}{|c|c|c|c|c|c|c|c|c|c|c|c|c|}
\hline & $C_{1}$ & $C_{2}$ & $C_{3}$ & $C_{4}$ & $C_{5}$ & $C_{6}$ & $C_{7}$ & $C_{8}$ & $C_{9}$ & $C_{10}$ & $C_{11}$ & $C_{12}$ \\
\hline$e_{j}$ & 0.9369 & 0.9687 & 0.9172 & 0.9249 & 0.8877 & 0.9028 & 0.9147 & 0.9661 & 0.8627 & 0.9086 & 0.9369 & 0.9687 \\
\hline$\theta_{j}$ & 0.0707 & 0.0351 & 0.0927 & 0.0841 & 0.1258 & 0.1089 & 0.0956 & 0.0380 & 0.1538 & 0.1023 & 0.0707 & 0.0351 \\
\hline$\sigma_{j}$ & 0.0855 & 0.0707 & 0.0968 & 0.0904 & 0.0997 & 0.0991 & 0.0842 & 0.0610 & 0.0963 & 0.0782 & 0.0656 & 0.0726 \\
\hline
\end{tabular}

Application of Equation (9), the weight distribution coefficient of $\Delta \phi$ carries out the rule changes in the numerical interval of $0-1$ with the step of 0.1 , to determine the ratio coefficient between the entropy weight and the variation weight. In the light of $G=\sum_{m}^{i=1} \sum_{n}^{j=1} x_{i j} \bar{\varpi}_{j}$, the risk fuzzy evaluation result could be reasoned for all the observed units under the different weight distribution coefficient of $\Delta \phi$, as shown in Tab.3.

The next step is to reckon the credibility of all the observed units with Equation (13), namely the value of $\mathrm{Cre}_{1}, \mathrm{Cre}_{2}, \mathrm{Cre}_{3}, \mathrm{Cre}_{4}, \mathrm{Cre}_{5}$ and $\mathrm{Cre}_{6}$ as 0.9379, 1.0, 0.9079, 0.9644, 0.9136 and 0.9879.

According to Equation (11), the overall risk environment is evaluated with the fuzzy comprehensive method under the various conditions of $\Delta \phi$, as the row vector in Tab.3. Obviously, under the different value changes of $\Delta \phi$, the overal risk degree changes in the numerical interval of 0.6387 - 0.6389 , with the relative constant risk evaluation for the certain earthquake-damaged environment in the mountain underground structures. Therefore, following the membership rule, this 
earthquake-damaged risk environment should be judged in the grade of $V_{\text {II }}$, namely the worse risk grade.

\begin{tabular}{|c|c|c|c|c|c|c|c|c|c|c|c|}
\hline & $\Delta \phi=0$ & $\Delta \phi=0.1$ & $\Delta \phi=0.2$ & $\Delta \phi=0.3$ & $\Delta \phi=0.4$ & $\Delta \phi=0.5$ & $\Delta \phi=0.6$ & $\Delta \phi=0.7$ & $\Delta \phi=0.8$ & $\Delta \phi=0.9$ & $\Delta \phi=1.0$ \\
\hline$u_{1}$ & 0.5438 & 0.5420 & 0.5402 & 0.5384 & 0.5366 & 0.5347 & 0.5329 & 0.5311 & 0.5293 & 0.5275 & 0.5256 \\
\hline$u_{2}$ & 0.6652 & 0.6673 & 0.6694 & 0.6715 & 0.6736 & 0.6757 & 0.6778 & 0.6799 & 0.6820 & 0.6841 & 0.6862 \\
\hline$u_{3}$ & 0.5511 & 0.5516 & 0.5521 & 0.5526 & 0.5530 & 0.5535 & 0.5540 & 0.5545 & 0.5550 & 0.5555 & 0.5560 \\
\hline$u_{4}$ & 0.5522 & 0.5522 & 0.5522 & 0.5521 & 0.5521 & 0.5521 & 0.5520 & 0.5520 & 0.5519 & 0.5519 & 0.5519 \\
\hline$u_{5}$ & 0.4313 & 0.4289 & 0.4266 & 0.4242 & 0.4219 & 0.4195 & 0.4172 & 0.4148 & 0.4124 & 0.4101 & 0.4077 \\
\hline$u_{6}$ & 0.5985 & 0.6000 & 0.6015 & 0.6030 & 0.6045 & 0.6059 & 0.6074 & 0.6089 & 0.6104 & 0.6119 & 0.6134 \\
\hline$u^{*}$ & 0.6387 & 0.6387 & 0.6387 & 0.6388 & 0.6388 & 0.6388 & 0.6388 & 0.6389 & 0.6389 & 0.6389 & 0.6389 \\
\hline
\end{tabular}

\section{Conclusion}

(1) Following the logical judgment process of "Specific - Abstract - Specific", the index system is established for the earthquake-damaged environment hazard evaluation operation, and the risk condition is divided into 5 grades with the certain fuzzy membership degree.

(2)Through discriminating the index entropy weight and the variation information in the fuzzy decision-making matrix for the earthquake-damaged underground structure environment, one weighting method is proposed with the integration of the index entropy weight and the variation information, and one method is given to dynamic revise and check the index weights with the index weight distribution coefficient.

(3) Application of Fuzzy Comprehensive Evaluation Method, the earthquake-damaged environment hazard comprehensive evaluation model is established, which settles the risk decision-making problem. Under the the dynamic revised index weights, the hazard evaluation coefficient could be reasoned for all the observed units.

(4) Under the similarity support degree within the risk evidence body, the risk decision-making matrix is synthesized with the similarity vector, and the risk degree of the whole earthquake-damaged environment is analyzed, which obtains the risk coefficient and grade about this certain evaluation issue.

\section{Acknowledgements}

This work was supported by the National Natural Science Foundation of China (Grant No.51308541) and the Natural Science Foundation of Jiangsu Province (Grant No.BK20130066).

\section{References}

[1]J.P. Wang, H. Taheri. Seismic Hazard Assessment of the Tehran Region. Natural Hazards Review, Vo.15-2 (2014), p.121-127.

[2]F.S. Wang, H.J. Wu, H.J. Zhang. Study on Risk Management in Rapid Repair Operations for Earthquake-damaged Underground Engineering. Chinese Journal of Systems Science, Vol.20-1 (2012) , p.75-79.

[3]J.W. Zhang, Z.R. Mei, X.J. Quan. Failure characteristics and influencing factors of highway tunnel damage due to earthquakes. Electronic Journal of Geotechnical Engineering, Vo.18E (2013), p.973-986. 
[4]W. Wang, J.Y. Su, D.H. Ma. Risk degree of earthquake secondary geological hazards evaluation method based on parameters optimization of SVC. Journal of Beijing University of Technology, Vol.38-10 (2012), p.1498-1503.

[5]J.L. Jin, Y.M. Wei, L.L. Zou,et al. Risk evaluation of China's natural disaster systems: an approach based on triangular fuzzy numbers and stochastic simulation. Natural Hazards, Vol.62-1 (2012), p.129-139.

[6]S.S. Shi, S.C. Li, LP. Li, et al. Advance optimized classification and application of surrounding rock based on fuzzy analytic hierarchy process and Tunnel Seismic Prediction. Automation in Construction, Vol.37(2014), p.217 - 222.

[7]S.T. Lu, S.H. Yu, D.S. Chang,et al. Using Fuzzy Multiple Criteria Decision-Making Approach for Assessing the Risk of Railway Reconstruction Project in Taiwan. The Scientific World Journal, Vol.2014, Article ID 239793.

[8]C.P. Mu, H.K. Huang, S.F. Tian. Intrusion detection alert verification based on multi-level fuzzy comprehensive evaluation. Lecture Notes in Computer Science, Vol.38-1 (2005) , p.9-16.

[9]B.S. Liu, Y.H. Shen, W. Zhang, etal. An interval-valued intuitionistic fuzzy principa lcomponent analysis model-based method for complex multi-attribute large-group decision-making. European Journal of Operational Research, Vol.245(2015), p.209-225.

[10]A. Quteishat, C.P. Lim. A modified fuzzy min-max neural network with rule extraction and its application to fault detection and classification. Applied Soft Computing, Vol.8-2 (2008), p.985-995.

[11]H.C. Ren, Z.Z. Shen. Analysis on Earthquake Damage Forms and Affecting Factors of Underground Cavern. Applied Mechanics and Materials, Vol.204-208 (2012), p.2444-2448.

[12]B.R. Xu, R.L. Li, H.M. Wei, et al. Entropy Weight Fuzzy Comprehensive Evaluation on Failure Mode Criticality of Integrated Transmission System. Acta ArmamentarII, Vol.34-11（2013）, p.1380-1386.

[13]F.S. Wang, H.J. Zhang, Y. Cao, et al. A Risk Early-warning Method in Earthquake Emergency Response based on Integrated Information Entropy and Matter-Element Model. International Journal of Intelligent Control and Systems, Vol.16-1（2011），pp.1-7.

[14]Y.C. Cao, F.L. Liu. Reliability analysis of foundation bearing capacity based on combined coefficients of variation. Rock and Soil Mechanics, Vol.35-7 (2014) , p.1950-1957.

[15]Y.W. Shen, X.T. Peng, T.Q. Shi, et al. A Grey Comprehensive Evaluation Method of Power Quality Based on Optimal Combination Weight. Chinese Journal of Automation of Electric Power Systems, Vol.36-10 (2012) , p.67-73.

[16]J. Liu, Y.C. Long. Prospect building method based on Dempster-Shafer theory DU Yuan-wei. Control and Decision, Vol.30-4 (2015) , p.759-763. 